Research Article

\title{
On the Emergence of the Coulomb Forces in Quantum Electrodynamics
}

\author{
Jan Naudts \\ Physics Department, University of Antwerp, Universiteitsplein 1, 2610 Antwerpen, Belgium \\ Correspondence should be addressed to Jan Naudts; jan.naudts@uantwerpen.be
}

Received 6 April 2017; Accepted 16 May 2017; Published 6 June 2017

Academic Editor: Thierry Grandou

Copyright (C) 2017 Jan Naudts. This is an open access article distributed under the Creative Commons Attribution License, which permits unrestricted use, distribution, and reproduction in any medium, provided the original work is properly cited. The publication of this article was funded by SCOAP .

\begin{abstract}
A simple transformation of field variables eliminates Coulomb forces from the theory of quantum electrodynamics. This suggests that Coulomb forces may be an emergent phenomenon rather than being fundamental. This possibility is investigated in the context of reducible quantum electrodynamics. It is shown that states exist which bind free photon and free electron fields. The binding energy peaks in the long-wavelength limit. This makes it plausible that Coulomb forces result from the interaction of the electron/positron field with long-wavelength transversely polarized photons.
\end{abstract}

\section{Introduction}

Quantum electrodynamics (QED), although very successful, is not a mathematically rigorous theory. Several difficulties have to be resolved or circumvented during the search for a consistent theory. One of them is the excess number of degrees of freedom in the description of the electromagnetic fields. In this context, the approach of Gupta [1] and Bleuler [2] is well known. An alternative is found in the work of Creutz [3]. He uses a unitary transformation which yields wave functions which do not undergo Coulomb forces. In quantum chromodynamics, the light cone gauge is used because then only transverse gluons remain [4]. In the present work, the assumption is that only transverse photons exist and that the number of degrees of freedom of the electromagnetic field is two. The argument of Creutz is used in opposite direction to define field operators which satisfy the Maxwell equations in presence of Coulomb forces.

Recently, Verlinde $[5,6]$ developed a cosmological theory in which gravity forces are emergent forces, in the sense that they are produced by other, more fundamental, forces of nature. A similar statement is investigated here for the role of Coulomb forces in QED. Not only are the degrees of freedom of the electromagnetic field limited to two, but also the Hamiltonian does not contain a Coulomb potential.
Further mathematical problems of QED disappear if one abandons the axiom that representations of the canonical commutation and anticommutation relations must be irreducible. This reducible QED is studied in the work of Czachor and collaborators (see [7-9] and references given in these papers). This formalism can be simplified along the lines worked out by the author in a series of papers [10-12].

The reduction of the representation involves an integration over three-dimensional wave vectors. At fixed wave vector, the system is purely quantum mechanical and consists of a pair of harmonic oscillators at each position in space-time to cover transversely polarized photons. A 16-dimensional Hilbert space describes the different states of an electronpositron field.

The mediation of Coulomb forces by transverse photons can be understood by analogy with the polaron problem of Solid State Physics. The polaron [13] is a state binding an electron with quantized lattice vibrations. In a polarized medium, the electric field of the electron is completely screened by redistribution of charges in its vicinity. The remaining interactions between electron and medium result in an attractive force between pairs of polarons. In some situations, it is strong enough to form bipolarons.

The present work shows that a free electron field can form a bound state with transversely polarized photons to form 
a dressed electron field. By analogy with the polaron case, one can then expect that dressed electrons interact with each other and that Coulomb forces can be explained in this way.

The next section introduces a transformation of field variables which adds Coulomb forces to transverse photons interacting with a charge field. The new field variables form what is called here the emergent picture of QED. They satisfy the full Maxwell equations. Section 3 highlights some aspects of reducible QED. Section 4 discusses the proof of the existence of bound states of transverse photons in interaction with the electron field. The final section gives a short discussion of what actually has been achieved.

\section{The Emergent Picture}

In [3], the temporal gauge, also called Weyl gauge or Hamiltonian gauge, is used. A unitary transformation $\widehat{V}$ is defined by a generator $\widehat{T}(\mathbf{x})$ through

$$
\widehat{V}=\exp \left(i \int \mathrm{d}^{3} \mathbf{x} \widehat{T}(\mathbf{x})\right) .
$$

The generator is of the form

$$
\widehat{T}(\mathbf{x})=\frac{q}{4 \pi} \int \mathrm{d}^{3} \mathbf{y} \widehat{\mathbf{A}}(x) \cdot \frac{\mathbf{x}-\mathbf{y}}{|\mathbf{x}-\mathbf{y}|^{3}} \widehat{j}_{0}(\mathbf{y}) .
$$

Here, $q$ is the elementary unit of charge. Bold characters are used to indicate 3-vectors. The result of [3], in the context of standard QED, is that

$$
\widehat{V} \nabla \cdot \widehat{\mathbf{E}} \widehat{V}^{-1}=\nabla \cdot \widehat{\mathbf{E}}-q \widehat{j_{0}}
$$

where $\widehat{\mathbf{E}}(x)$ are the electric field operators. If they satisfy Gauss's law in the presence of a charge distribution $\widehat{j}_{0}(\mathbf{x})$, then $\widehat{V} \mathbf{E} \widehat{V}^{-1}$ satisfies Gauss's law in absence of charges.

In the present work, the trick of [3] is directly applied to define new electric field operators:

$$
\begin{aligned}
\widehat{E}_{\alpha}^{\prime \prime}(x) & \\
= & \widehat{E}_{\alpha}^{\prime}(x) \\
& \quad+\frac{\mu_{0} c}{4 \pi} \frac{\partial}{\partial x^{\alpha}} \int \mathrm{d} \mathbf{y} \frac{1}{|\mathbf{x}-\mathbf{y}|} \widehat{U}\left(-x^{0}\right) \widehat{j}^{0}(\mathbf{y}, 0) \widehat{U}\left(x^{0}\right) .
\end{aligned}
$$

Here, $\widehat{U}\left(x^{0}\right)=\exp \left(-i x^{0} \widehat{H} / \hbar c\right)$ is the time evolution of the interacting system. The new operators are marked with a double prime to distinguish them from the operators of the noninteracting system and those of the interacting system. The latter are denoted with a single prime. One verifies immediately that Gauss' law is satisfied:

$$
\sum_{\alpha} \frac{\partial}{\partial x^{\alpha}} \widehat{E}_{\alpha}^{\prime \prime}(x)=-\mu_{0} c \hat{j}^{0 \prime}(x) .
$$

The second term of (4) is the Coulomb contribution to the electric field. The curl of this term vanishes. Hence, it is obvious to take

$$
\widehat{B}_{\alpha}^{\prime \prime}(x) \equiv \widehat{B}_{\alpha}^{\prime}(x)
$$

This implies the second of the four equations of Maxwell, stating that the divergence of $\widehat{B}_{\alpha}^{\prime \prime}(x)$ vanishes. Also the fourth equation, absence of magnetic charges, follows immediately because $\widehat{E}^{\prime \prime}(x)$ and $\widehat{E}^{\prime}(x)$ have the same curl. Faraday's law remains to be written as

$$
\left(\nabla \times \widehat{B}^{\prime \prime}(x)\right)_{\alpha}-\frac{1}{c} \frac{\partial}{\partial x^{0}} \widehat{E}_{\alpha}^{\prime \prime}(x)=-\mu_{0} \hat{j}_{\alpha}^{\prime \prime}(x)
$$

with

$$
\hat{j}_{\alpha}^{\prime \prime}(x)=-\frac{1}{\mu_{0} c} \frac{\partial}{\partial x^{0}}\left(\widehat{E}_{\alpha}^{\prime \prime}(x)-\widehat{E}_{\alpha}^{\prime}(x)\right) .
$$

Finally, take $\hat{j}_{0}^{\prime \prime}(x)=\hat{j}_{0}^{\prime}(x)$. A short calculation shows that the newly defined current operators $\hat{j}_{\mu}^{\prime \prime}(x)$ satisfy the continuity equation.

One concludes that a formalism of QED is possible which does not postulate the existence of longitudinal or scalar photons. Two pictures coexist: the original Heisenberg picture and what is called here the emergent picture. In both pictures, the time evolution of all operators is the same, but the definition of the electromagnetic field operators differs. In the original description, only transversely polarized photons exist. On the other hand, the field operators of the emergent picture satisfy the full Maxwell equations, including Coulomb forces.

\section{Reducible QED}

A dominant characteristic of reducible QED, in the version used here, is that many expressions look familiar from standard QED, except that integrations over the wave vector are missing. They are postponed to the evaluation of quantum expectation values. As a consequence, field operators depend on both position $x$ in spacetime and wave vector $\mathbf{k}$ in $\mathbb{R}^{3}$. For instance, the electromagnetic potential operators are defined by

$$
\begin{aligned}
\widehat{A}_{\alpha}(x)= & \frac{1}{2} \lambda \varepsilon_{\alpha}^{(\mathrm{H})}\left(\mathbf{k}^{\mathrm{ph}}\right)\left[e^{-i k_{\mu}^{\mathrm{ph}} x^{\mu}} \widehat{a}_{\mathrm{H}}+e^{i k_{\mu}^{\mathrm{ph}} x^{\mu}} \widehat{a}_{\mathrm{H}}^{\dagger}\right] \\
& +\frac{1}{2} \lambda \varepsilon_{\alpha}^{(\mathrm{V})}\left(\mathbf{k}^{\mathrm{ph}}\right)\left[e^{-i k_{\mu}^{\mathrm{ph}} x^{\mu}} \widehat{a}_{\mathrm{V}}+e^{i k_{\mu}^{\mathrm{ph}} x^{\mu}} \hat{a}_{\mathrm{V}}^{\dagger}\right] .
\end{aligned}
$$

Here, $\widehat{a}_{\mathrm{H}}, \widehat{a}_{\mathrm{H}}^{\dagger}, \widehat{a}_{\mathrm{V}}$, and $\hat{a}_{\mathrm{V}}^{\dagger}$ are the creation and annihilation operators of horizontally, respectively, vertically polarized photons. The dispersion relation of the photon is $k_{0}^{\mathrm{ph}}=\left|\mathbf{k}^{\mathrm{ph}}\right|$ as usual. $\varepsilon_{\alpha}^{(\mathrm{H})}\left(\mathbf{k}^{\mathrm{ph}}\right)$ and $\varepsilon_{\alpha}^{(\mathrm{V})}\left(\mathbf{k}^{\mathrm{ph}}\right)$ are polarization vectors. The parameter $\lambda$ is introduced for dimensional reasons.

A consequence of the missing integration over the wave vector is that equal-time fields become noncommutative. For instance, a calculation starting from (9) shows that

$$
\begin{aligned}
& {\left[\widehat{A}_{\alpha}(\mathbf{x}, 0), \widehat{A}_{\beta}(\mathbf{y}, 0)\right]_{-}} \\
& \quad=\frac{i \lambda^{2}}{2} F_{\alpha, \beta}\left(\mathbf{k}^{\mathrm{ph}}\right) \sin \left(\mathbf{k}^{\mathrm{ph}} \cdot(\mathbf{x}-\mathbf{y})\right),
\end{aligned}
$$

where $F\left(\mathbf{k}^{\mathrm{ph}}\right)$ projects onto the plane orthogonal to $\mathbf{k}^{\mathrm{ph}}$. If the integration over $\mathbf{k}^{\mathrm{ph}}$ is executed, then the standard result of vanishing equal-time commutators follows. 
Another feature of the theory is that wave functions are properly normalized for each wave vector separately. For instance, if $\psi$ describes an electron/positron field, then $\psi_{\mathbf{k}}$ is a wave function in the $\mathbf{k t h}$ Fock space and satisfies $\left\langle\psi_{\mathbf{k}} \mid \psi_{\mathbf{k}}\right\rangle=$ 1 for each value of $\mathbf{k}$. Superpositions of wave functions with different wave vector are allowed. The general wave function is therefore of the form

$$
\psi_{\mathbf{k}}=\sum_{X \subset\{1,2,3,4\}} \sqrt{\rho_{X}(\mathbf{k})}|X\rangle \quad \text { for any } \mathbf{k} .
$$

The set $X$ selects one of the 16 possible states of an electron/positron field. The empty set $\emptyset$ refers to the vacuum state $|\emptyset\rangle$. Normalization requires that

$$
\sum_{X \subset\{1,2,3,4\}} \rho_{X}(\mathbf{k})=1 \quad \text { for any } \mathbf{k} .
$$

Ultraviolet divergences are avoided by requiring that $\rho_{X}(\mathbf{k})$ with $X \neq \emptyset$ vanishes for large values of $|\mathbf{k}|$. Similarly, the general wave function of the free electromagnetic field is of the form

$$
\psi_{\mathbf{k}^{\mathrm{ph}}}=\sum_{m, n=0}^{\infty} \sqrt{\rho_{m, n}\left(\mathbf{k}^{\mathrm{ph}}\right)} e^{i \phi(m, n)}|m, n\rangle .
$$

Normalization requires that

$$
\sum_{m, n=0}^{\infty} \rho_{m, n}\left(\mathbf{k}^{\mathrm{ph}}\right)=1 \quad \text { for any } \mathbf{k}^{\mathrm{ph}} .
$$

The Dirac currents $\hat{j}^{\mu}(x)$ are defined in terms of Dirac spinors $\widehat{\psi}_{r}(x)$ which satisfy a free Dirac equation (see [11] for details). The Dirac equation is only used to define currents $\hat{j}^{\mu}(x)$ in absence of interaction with the electromagnetic fields. No interacting Dirac equation is considered. Instead, the interactions between the free field operators are described by the usual interaction Hamiltonian in a Heisenberg picture. See (17) below.

\section{Bound States}

Let $\hat{b}_{\uparrow}^{\dagger}$ denote the creation operator for an electron with spin up. An example of a realistic electron field is described by

$$
\psi^{\mathrm{el}}=e^{i \chi(\mathbf{k})} \sqrt{1-\rho^{\mathrm{el}}(\mathbf{k})}|\emptyset\rangle+\sqrt{\rho^{\mathrm{el}}(\mathbf{k})} \hat{b}_{\uparrow}^{\dagger}|\emptyset\rangle .
$$

Similarly, a realistic wave function for a horizontally polarized photon is

$$
\psi^{\mathrm{ph}}=\sqrt{\rho^{\mathrm{ph}}\left(\mathbf{k}^{\mathrm{ph}}\right)} \widehat{a}_{\mathrm{H}}^{\dagger}|\emptyset\rangle+\sqrt{1-\rho^{\mathrm{ph}}\left(\mathbf{k}^{\mathrm{ph}}\right)}|\emptyset\rangle .
$$

The Hamiltonian is the usual one, with interaction part

$$
\widehat{H}^{\mathrm{I}}=\int \mathrm{d} \mathbf{x} \widehat{j}^{\mu}(\mathbf{x}, 0) \widehat{A}_{\mu}(\mathbf{x}, 0) .
$$

Assume that $\rho^{\mathrm{el}}\left(-\mathbf{k}^{\mathrm{el}}\right)=\rho^{\mathrm{el}}\left(\mathbf{k}^{\mathrm{el}}\right)$. Then the average interaction energy of a product state $\psi=\psi^{\mathrm{ph}} \psi^{\mathrm{el}}$ vanishes for symmetry reasons. However, there exist entangled wave functions which lower the total energy.

Choose, for instance, an entangled wave function of the form

$$
\begin{aligned}
& \psi_{\mathbf{k}^{\mathrm{ph}}, \mathbf{k}}= {\left[\tau\left(\mathbf{k}^{\mathrm{ph}}, \mathbf{k}\right) \hat{a}_{\mathrm{H}}^{\dagger}+1-\tau\left(\mathbf{k}^{\mathrm{ph}}, \mathbf{k}\right)\right] } \\
& \cdot \sqrt{\rho\left(\mathbf{k}^{\mathrm{ph}}\right) \rho^{\mathrm{el}}(\mathbf{k}) \hat{b}_{\uparrow}^{\dagger}}|\emptyset\rangle \\
&+\sqrt{1-\rho\left(\mathbf{k}^{\mathrm{ph}}\right) \rho^{\mathrm{el}}(\mathbf{k})}|\emptyset\rangle,
\end{aligned}
$$

where $\tau\left(\mathbf{k}^{\mathrm{ph}}, \mathbf{k}\right)$ equals either 1 or 0 . Assume in addition that the electron density $\rho^{\mathrm{el}}(\mathbf{k})$ has a Gaussian shape. Then an explicit calculation shows that the binding energy peaks for long-wavelength horizontally polarized photons with wave vector $\mathbf{k}^{\mathrm{ph}}$ in principle direction 1 (see [12] for detailed calculations). Moreover, the total energy is lower than that of the free electron. One concludes that there exist states binding an electron field and a transversely polarized photon field.

\section{Discussion}

In a theory with only transverse photons and no Coulomb forces, a simple transformation of the field variables, given by (4), introduces new variables which satisfy the full Maxwell equations. The new variables form what is called here the emergent picture of QED. The time evolution of operators is the same in the emergent picture as in the original Heisenberg picture. Hence, one can avoid introducing Coulomb forces if one does not want to have them.

Is this transformation more than a mathematical trick? A plausible explanation of the physics behind this transformation is that long-wavelength transverse photons produce effective forces between different parts of the electron/positron field. The expectation is that these effective forces coincide with what is known as Coulomb forces.

Investigation of the scenario sketched above starts with a mathematical proof that transverse photons do interact with the electron/positron field and even can form bound states. This proof is given in the context of reducible QED because this formalism allows for a mathematically rigorous treatment. Details of the proof are found elsewhere [12]. The next thing to do is an analysis of the time evolution of these bound states. This analysis is still missing.

\section{Conflicts of Interest}

The author declares that there are no conflicts of interest regarding the publication of this paper.

\section{References}

[1] S. N. Gupta, "Theory of longitudinal photons in quantum electrodynamics," Proceedings of the Physical Society. Section A, vol. 63, no. 7, article no. 301, pp. 681-691, 1950.

[2] K. Bleuler, "Eine neue methode zur Behandlung der longitudinalen und skalaren photonen," Helvetica Physica Acta, vol. 23, pp. 567-586, 1950. 
[3] M. Creutz, "Quantum electrodynamics in the temporal gauge," Annals of Physics, vol. 117, no. 2, pp. 471-483, 1979.

[4] S. J. Brodsky, H.-C. Pauli, and S. S. Pinsky, "Quantum chromodynamics and other field theories on the light cone," Physics Reports. A Review Section of Physics Letters, vol. 301, no. 4-6, pp. 299-486, 1998.

[5] E. Verlinde, "On the origin of gravity and the laws of Newton," Journal of High Energy Physics, vol. 4, p. 29, 2011.

[6] E. P. Verlinde, "Emergent Gravity and the Dark Universe," https://arxiv.org/abs/1611.02269.

[7] M. Czachor, "Non-canonical quantum optics," Journal of Physics. A. Mathematical and General, vol. 33, no. 45, pp. 80818103, 2000.

[8] M. Czachor and J. Naudts, "Regularization as quantization in reducible representations of CCR," International Journal of Theoretical Physics, vol. 46, no. 1, pp. 73-104, 2007.

[9] M. Czachor and K. Wrzask, "Automatic regularization by quantization in reducible representations of CCR: Point-form quantum optics with classical sources," International Journal of Theoretical Physics, vol. 48, no. 9, pp. 2511-2549, 2009.

[10] J. Naudts, "Reducible Quantum Electrodynamics. I. The Quantum Dimension of the Electromagnetic Field," https:// arxiv.org/abs/1506.00098.

[11] J. Naudts, "Reducible Quantum Electrodynamics. II. The charged states of the vacuum," https://arxiv.org/abs/1510.02640.

[12] J. Naudts, "Reducible Quantum Electrodynamics. III. The emergence of the Coulomb forces," https://arxiv.org/abs/1703.04952.

[13] J. T. Devreese and A. S. Alexandrov, "Fröhlich polaron and bipolaron: recent developments," Reports on Progress in Physics, vol. 72, no. 6, p. 066501, 2009. 

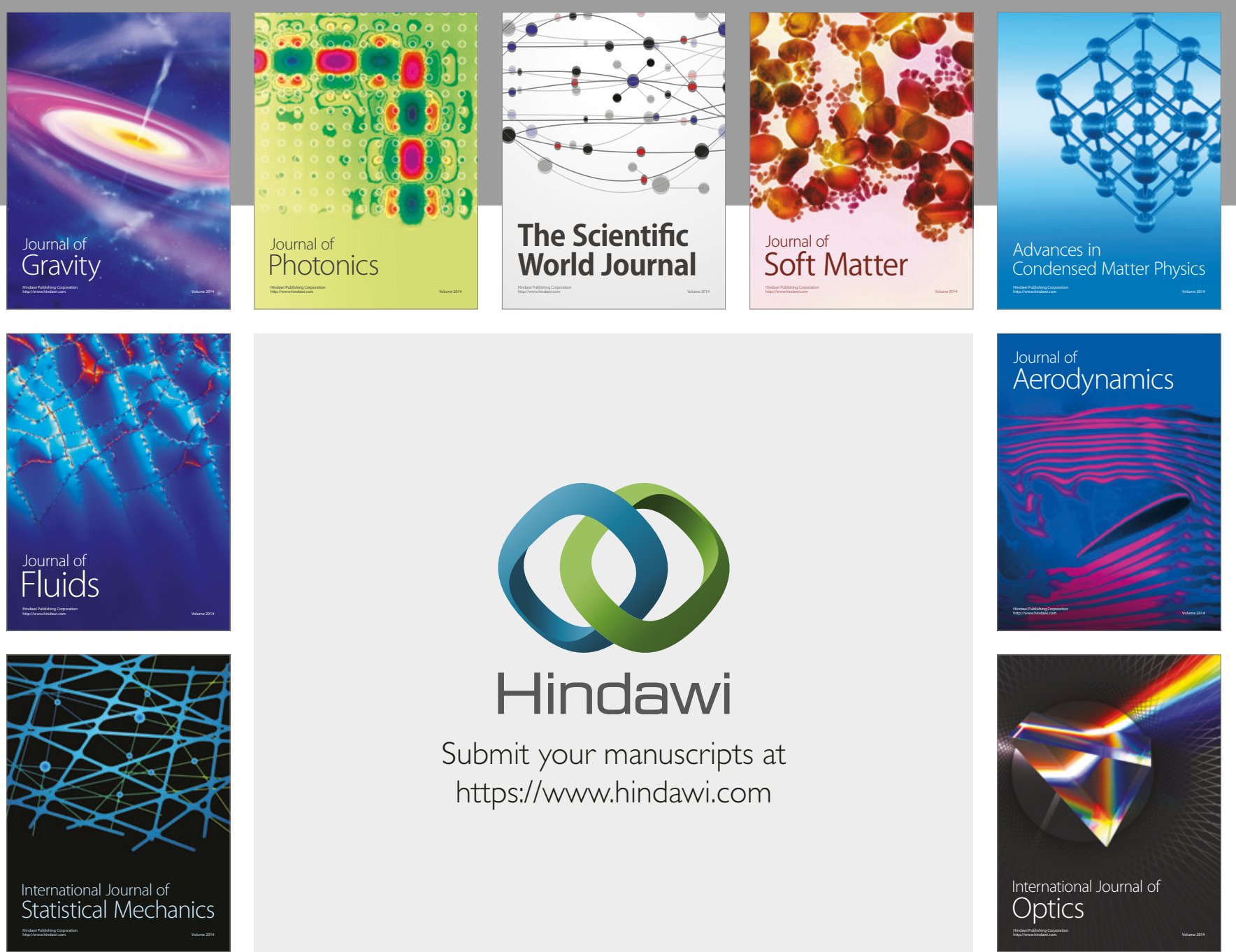

Submit your manuscripts at

https://www.hindawi.com
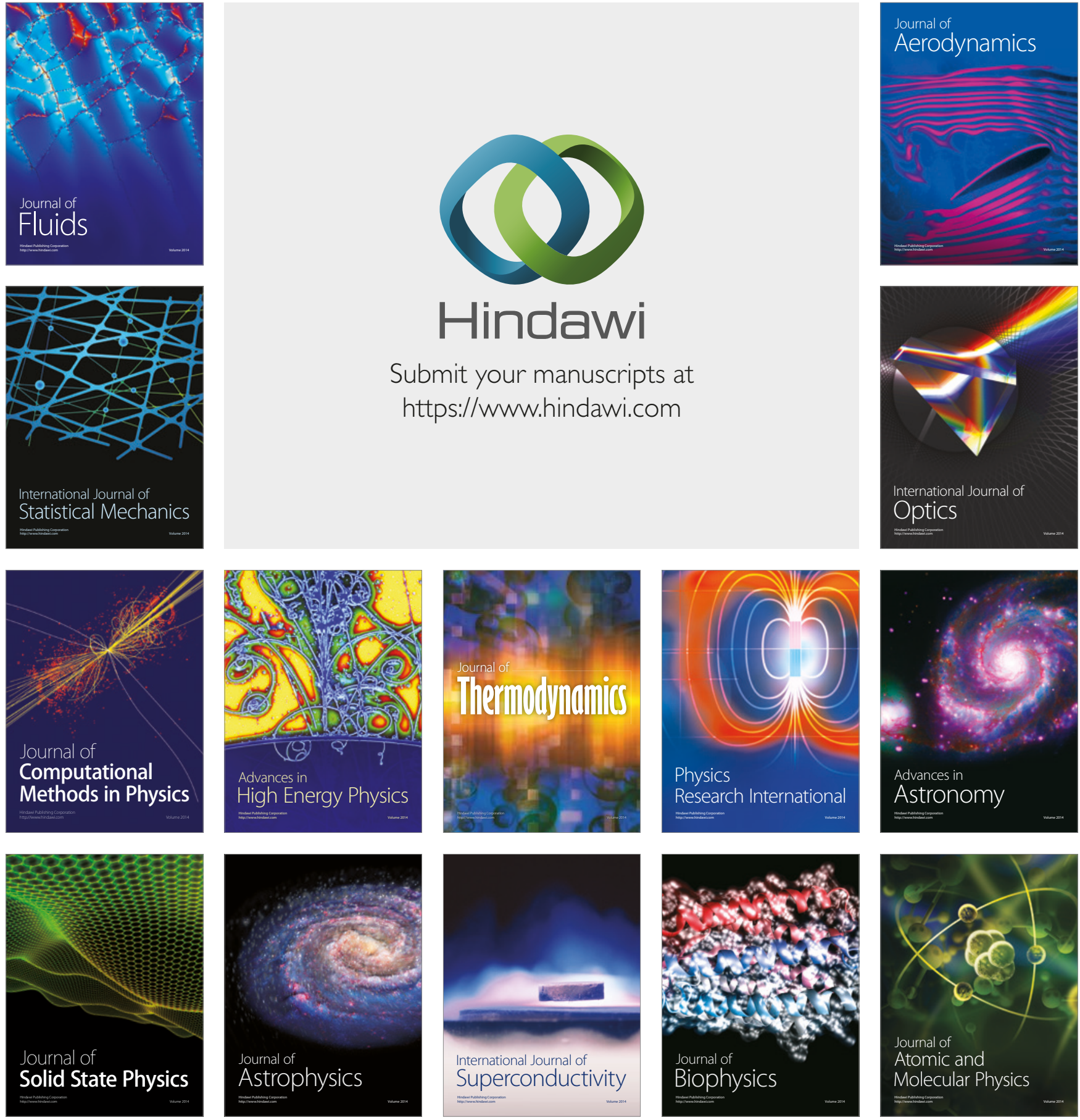